\title{
Effectiveness of Safinamide over Mood in Parkinson's Disease Patients: Secondary Analysis of the Open-label Study SAFINONMOTOR
}

\author{
Carmen M. Labandeira $\cdot$ Maria G. Alonso Losada $\cdot$ Rosa Yáñez Baña \\ Maria I. Cimas Hernando · Iria Cabo López · Jose M. Paz González • \\ Maria J. Gonzalez Palmás · Cristina Martínez Miró • Diego Santos García (D)
}

Received: June 25, 2021 / Accepted: July 23, 2021 / Published online: September 15, 2021

(C) The Author(s) 2021

\begin{abstract}
Introduction: Mood disorders are frequent in Parkinson's disease (PD) and a favorable effect of safinamide on mood has been observed. We aimed to analyze the effectiveness of safinamide on mood as a secondary objective from the SAFINONMOTOR (an open-label study of the effectiveness of SAFInamide on NON-MOTOR symptoms in patients with Parkinson's disease) study.
\end{abstract}

Supplementary Information The online version contains supplementary material available at https:// doi.org/10.1007/s12325-021-01873-w.

C. M. Labandeira - M. G. Alonso Losada CHUVI, Complejo Hospitalario Universitario de Vigo, Vigo, Spain

R. Yáñez Baña

CHUO, Complejo Hospitalario Universitario de Ourense, Ourense, Spain

M. I. Cimas Hernando

Hospital de Povisa, Vigo, Spain

I. Cabo López · M. J. Gonzalez Palmás

CHOP, Complejo Hospitalario Universitario de

Pontevedra, Pontevedra, Spain

J. M. Paz González · C. Martínez Miró .

D. Santos García ( $\square)$

Department of Clinical Neurology, CHUAC,

Complejo Hospitalario Universitario de A Coruña,

As Xubias 84, 15006 A Coruña, Spain

e-mail: diegosangar@yahoo.es
Methods: SAFINONMOTOR is a prospective open-label single-arm study conducted in five centers from Spain. Patients with PD were required to have at baseline a Non-Motor Symptoms Scale (NMSS) total score of at least 40. In this analysis, the changes from V1 (baseline) to V4 (6 months \pm 1 month) in the BDI-II (Beck Depression Inventory-II), NMSS mood/apathy domain, and PDQ-39 (Parkinson's Disease Questionnaire-39) emotional well-being domain were analyzed. Depression was identified and classified (DSM-IV and Judd criteria) at baseline and at the end of follow-up as major depression (MD), minor depression (mD), subthreshold depression (subD), and non-depression (nonD).

Results: Fifty patients with PD were included (age $\quad 68.5 \pm 9.12$ years; $58 \% \quad$ women; $6.4 \pm 5.1$ years from diagnosis) and 44 patients (88\%) completed the follow-up at 6 months. The BDI-II total score was reduced by $35.9 \%$ (from $15.88 \pm 10.46$ at V1 to $10.18 \pm 6.76$ at $\mathrm{V} 4 ; p<0.0001)$. A significant decrease in the NMSS mood/apathy domain and PDQ-39 emotional well-being domain was observed as well $(p<0.0001)$. At baseline, $52 \%$ of the patients presented $\mathrm{MD}, 34 \% \mathrm{mD}, 12 \%$ subD, and $2 \%$ nonD whereas at $\mathrm{V} 4$ the percentages were $31.8 \%, 34.1 \%, 22.7 \%$, and $11.4 \%$, respectively $(p=0.029)$.

Conclusions: Safinamide improves mood in patients with PD at 6 months. 
Keywords: Safinamide; Non-motor symptoms; Parkinson's disease; Depression; Mood

\section{Key Summary Points}

Why carry out this study?

Non-motor symptoms (NMS) are frequent in patients with Parkinson's disease (PD). Mood disorders can be present in up to $50 \%$ of patients with PD.

Some studies suggest a possible benefit of patients with PD after treatment with safinamide, a drug used as an add-on treatment for PD with "off" episodes, on global NMS burden and in some NMS in particular such as sleep, pain, mood, or urinary symptoms.

In this analysis, a secondary objective of the open-label study SAFINONMOTOR, we evaluated in detail the change in mood throughout the 6-month follow-up in patients with PD treated with safinamide.

\section{What was learned from this study?}

Safinamide improved mood in patients with PD with severe or very severe nonmotor symptoms burden.

Safinamide showed a sustained significant benefit on BDI-II (Beck Depression Inventory-II), NMSS (Non-Motor Symptoms Scale) mood/apathy domain, and the PDQ-39 (Parkinson's Disease Questionnaire-39) emotional well-being scores at 6 months.

The benefit in treating mood was observed with both doses, $50 \mathrm{mg}$ at 1 month and $100 \mathrm{mg}$ at 6 months.

Study limitations included the sample size, not having a placebo arm, and inclusion of patients with PD with severe or very severe NMS burden. Further studies including double-blind and longer follow-ups are required to analyze the role of safinamide in the regulation of mood in PD.

\section{INTRODUCTION}

Parkinson's disease (PD) is the second most common neurodegenerative disease, involving both motor and non-motor symptoms (NMS). Depression is a very frequent NMS that seriously affects quality of life (QoL) in PD, even in subclinical patients [1]. Treatment of mood disorders in PD is a challenging issue not only because of the poor response to medications in many patients but also the risk of adverse events and drug interactions [2]. Safinamide is a highly selective and reversible inhibitor of monoamine oxidase B (MAOB-I), but it can also restrain glutamate release by selectively modulating sodium channel blockade and calcium channels. This combined mechanism has been suggested to improve NMS by dopaminergic and non-dopaminergic effects. Safinamide is effective in the treatment of motor fluctuations [3] and NMS [4]. With regard to mood, very recently, Peña et al. [5] observed in a retrospective study conducted in 82 patients with PD a significant improvement in the Hamilton Depression Rating Scale 3 months after starting with safinamide and suggested that safinamide could be useful for treating depression in PD. Previously, it was reported that safinamide, compared to placebo, significantly improved the PDQ-39 "Emotional well-being" domain after 6 months and 2 years, as well as the GRID Hamilton Rating Scale for Depression [6].

We observed very recently an improvement in global NMS burden in 50 patients with PD from the SAFINONMOTOR study (an open-label study of the effectiveness of SAFInamide on NON-MOTOR symptoms in patients with Parkinson's disease) [7]. In this analysis, a secondary objective of the SAFINONMOTOR study, we evaluated in detail the change in mood throughout the 6-month follow-up in patients with PD treated with safinamide.

\section{METHODS}

SAFINONMOTOR is an observational (phase IV), prospective, open-label, follow-up multicenter study developed to analyze the effectiveness of 
safinamide on NMS in patients with PD [7]. The analysis of changes in mood was a secondary objective defined in the study protocol. Patients were enrolled according to the following inclusion criteria: (1) diagnosis of PD according to the UK Parkinson's Disease Society Brain Bank criteria [8]; (2) to have the indication of receiving safinamide according to the neurologist criteria in their clinical practice; (3) to have a Non-Motor Symptoms Scale (NMSS) total score at baseline of at least 40 ; (4) no dementia criteria with a MiniMental State Examination at baseline of at least 26) [9]; (5) to be older than 30 years old; (6) to wish to voluntarily participate and to sign a consent form. Exclusion criteria were (1) to be under MAOB-I (rasagiline or selegiline; a washout period of at least 2 week was allowed); (2) any other contraindication to be treated with safinamide according to product data; (3) incapacity to complete the questionnaires adequately; (4) other disabling concomitant neurological disease (stroke, severe head trauma, neurodegenerative disease, etc.); (5) other severe and disabling concomitant non-neurological disease (oncological, autoimmune, etc.); (6) expected impossibility of long-term follow-up; (7) patient who was participating in a clinical trial and/or other type of study. All the neurologists who participated in the study were experts on movement disorders.

Patients were evaluated at baseline before initiating treatment with safinamide. Follow-up visits were programmed after 1 month ( \pm 7 days) (V2), at 3 months ( \pm 15 days) (V3), and at 6 months ( \pm 15 days) (V4, end of the observational period). Safinamide was started at $50 \mathrm{mg}$ once daily and increased after 1 month at V2 from 50 to $100 \mathrm{mg}$, but in some cases (e.g., dyskinesia) the dose of $100 \mathrm{mg}$ could be introduced earlier or the dose could be kept at $50 \mathrm{mg} /$ day according to the criteria of the neurologist. Any other PD or non-PD medications during follow-up could not be changed unless the neurologist considered these changes absolutely necessary. All the changes including PD and not-PD-related medications and levodopa equivalent daily dose of levodopa were recorded.

Patients completed the Beck Depression Inventory (BDI)-II, NMSS, and 39-item
Parkinson's Disease Quality of Life Questionnaire (PDQ-39) in all visits. The mood/apathy domain of the NMSS was expressed as a percentage: (score/total score) $\times 100$. The PDQ-39 was expressed as a summary index [(PDQ-39SI): $($ score $/ 156) \times 100]$ and the emotional well-being domain was also calculated as a percentage. Depression was classified following DSM-IV and Judd criteria $[10,11]$ as major depression (MD), minor depression $(\mathrm{mD})$, subthreshold depression (subD), and non-depression (nonD). Specifically, in relation to items 1 (concerning sadness), 4 (loss of pleasure), 5 (guilty feelings), 9 (suicidal thoughts or wishes), 13 (indecisiveness), 15 (loss of energy), 16 (changes in sleeping pattern), 17 (irritability), and 18 (changes in appetite) of the BDI-II, depression was defined follows: MD, at least five symptoms with the presence of item 1 (feeling of sadness) and/or item 4 (anhedonia) (DSM-IV criteria); $\mathrm{mD}$, two to four symptoms with the presence of item 1 and/or item 4 (DSM-IV criteria); subD, from two to four symptoms without the presence of item 1 and item 4 (Judd criteria).

As a result of the possible influence of gender on depression in PD [12], we investigated possible gender differences in our cohort, comparing the change from V1 to V4 in the BDI-II, NMSS mood/apathy domain, and PDQ-39 emotional well-being domain scores between men and women. On the other hand and since the contribution of mood to QoL is greater in patients with longer disease duration [13], we also examined if disease duration or age could determine differences in mood results. Finally, correlations between changes in mood and other variables from V1 to V4 were analyzed.

\section{Ethics Statement}

The study was conducted according to the guidelines of the Declaration of Helsinki of 1964, and its later amendments. This study was approved by the by the Comite de Ética de la Investigación Clínica de Galicia from Spain (2018-052; 28/FEB/2019) and all participants gave their written informed consent before inclusion in the study. SAFINONMOTOR was classified by the AEMPS (Agencia Española del 
Medicamento y Productos Sanitarios) as a postauthorization prospective follow-up study with the code DSG-SAF-2018-01. The protocol and the statistical analysis plan are available on request.

\section{Data Analysis}

Data were processed using SPSS 20.0 for Windows. Continuous variables were expressed as the mean $\pm \mathrm{SD}$ or median and quartiles, depending on whether they were normally distributed. Relationships between variables were evaluated using the Student's $t$ test, the Mann-Whitney $U$ test, Spearman's or Pearson's correlation coefficient as appropriate (distribution for variables was verified by one-sample Kolmogorov-Smirnov test). The change from V1 to V4 in the BDI-II total score was the principal efficacy outcome variable in this analysis. Moreover, the changes from V1 to V4 in the NMSS mood/apathy domain and in the PDQ-39 emotional well-being domain scores, and in the percentage of patients with $\mathrm{MD}, \mathrm{mD}$, subD, and nonD, were analyzed, too. Wilcoxon's and marginal homogeneity tests were performed to testing the changes from V1 to V4. Spearman's or Pearson's correlation coefficient, as appropriate, was used for analyzing the relationship between the change from V1 to V4 in continuous variables. Correlations were considered weak for coefficient values of 0.29 or less, moderate for values between 0.30 and 0.59 , and strong for values of 0.60 or more. Analyses on efficacy variables were performed with the intention to treat (ITT) data set (all subjects who receive at least one pill of safinamide and had a baseline and treatment observation for the primary efficacy outcome measure). Values of $p<0.05$ were considered significant.

The safety data set consists of all subjects for whom the study device was initiated. Safety analyses was assessed by adverse events (AEs). All AEs were coded using the current version of the Medical Dictionary for Regulatory Activities (MedDRA). The number and percentage of subjects with treatment emergent AEs by MedDRA system organ class and preferred term, by severity, and by relationship to study treatment as assessed by the investigator were provided for overall subjects.

\section{RESULTS}

Between May 2019 and February 2020 a total of 50 patients were recruited to participate and 44 completed the follow-up at 6 months (age $68.5 \pm 9.12$ years; $58 \%$ women; $6.4 \pm 5.1$ years from diagnosis). Data about sociodemographic aspects, comorbidities, antiparkinsonian drugs, and other therapies are shown in Table 1 in the Supplementary Material. The mean time from diagnosis of PD was $6.39 \pm 5.06$ years. All patients except three were receiving levodopa, two patients were under levodopa/carbidopa infusion therapy, and none were receiving apomorphine or deep brain stimulation. At baseline (V1), 78\% (39/50) of the patients presented with motor fluctuations and 30\% (15/50) with dyskinesia.

At 6 months, 44 patients completed the follow-up (88\%) and a lower BDI-II score compared to baseline was observed in 34 out of 44 patients $(77.3 \%)$, the same score in 2 patients $(4.5 \%)$, and a higher score in 8 patients too $(18.2 \%)$. The mean BDI-II total score was reduced from V1 to V4 by $35.9 \%$ (from $15.88 \pm 10.46$ at $\mathrm{V} 1$ to $10.18 \pm 6.76$ at $\mathrm{V} 4$; $p<0.0001$ ) (Table 1 and Fig. 1 ). In the case of the NMSS mood/apathy domain and the PDQ39 emotional well-being domain, a reduction in the score from V1 to V4 of $57.9 \%$ (from $34.42 \pm 29.89$ at $\mathrm{V} 1$ to $14.49 \pm 19.63$ at V4; $p<0.0001$ ) and $40.6 \%$ (from $44.3 \pm 29.34$ at $\mathrm{V} 1$ to $26.33 \pm 23.01$ at $\mathrm{V} 4 ; p<0.0001)$, respectively, was observed (Table 1 and Figs. 2, 3). Compared to the score at V1, the change in the score at V2 and V3 for the BDI-II, NMSS mood/ apathy domain, and the PDQ-39 emotional well-being domain was significant too, but differences were not observed between the score from V2 and V3 to V4 for any of the scales (Table 2 and Figs. 1, 2, 3). With regard to the MDS-IV and Judd criteria, at the baseline visit, $2 \%(N=1)$ of patients presented nonD, $12 \%$ $(N=6)$ subD, $34 \%(N=17) \mathrm{mD}$, and $52 \%$ $(N=26)$ suffered MD. After 6 months, the 
Table 1 Change in the score of the BDI-II, NMSS mood/apathy domain, PDQ-39 emotional well-being domain, and other scales of the study from V1 (baseline) to V4 (6 months \pm 15 days)

\begin{tabular}{|c|c|c|c|c|c|c|}
\hline & V1 & $N(\mathbf{V} \mathbf{1})$ & V4 & $N(\mathbf{V} 4)$ & $\Delta \mathrm{V} 1-\mathrm{V} 4$ & $p$ \\
\hline \multicolumn{7}{|l|}{ Motor assessment } \\
\hline $\mathrm{H} \& \mathrm{Y}-\mathrm{OFF}$ & $2.5[2,3]$ & 46 & NA & & & \\
\hline $\mathrm{H} \& \mathrm{Y}-\mathrm{ON}$ & $2[2,2.5]$ & 49 & NA & & & \\
\hline UPDRS-III-ON & $24.63 \pm 9.12$ & 48 & $20.21 \pm 9.81$ & 39 & $-17.90 \%$ & 0.009 \\
\hline UPDRS-IV & $3.82 \pm 2.55$ & 50 & $2.82 \pm 2.38$ & 34 & $-125.40 \%$ & 0.188 \\
\hline FOGQ & $6.10 \pm 5.23$ & 48 & $5.68 \pm 4.96$ & 44 & $-16.90 \%$ & 0.24 \\
\hline \multicolumn{7}{|l|}{ Non motor assessment } \\
\hline NMSS total score & $97.48 \pm 43.7$ & 50 & $59.91 \pm 35.49$ & 44 & $-138.50 \%$ & $<0.0001$ \\
\hline Cardiovascular & $9.58 \pm 2.46$ & 50 & $6.72 \pm 11.94$ & 44 & $-129.90 \%$ & 0.268 \\
\hline Sleep/fatigue & $36.08 \pm 21.77$ & 50 & $23.15 \pm 18.12$ & 44 & $-135.80 \%$ & 0.002 \\
\hline Mood/apathy & $34.42 \pm 29.89$ & 50 & $14.49 \pm 19.63$ & 44 & $-157.90 \%$ & $<0.0001$ \\
\hline Perceptual symptoms & $4.33 \pm 8.67$ & 50 & $2.84 \pm 5.88$ & 44 & $-134.40 \%$ & 0.63 \\
\hline Attention/memory & $17.5 \pm 17.09$ & 50 & $13.32 \pm 18.19$ & 44 & $-123.90 \%$ & 0.026 \\
\hline Gastrointestinal symptoms & $19.61 \pm 18.01$ & 50 & $13.13 \pm 13.39$ & 44 & $-133.00 \%$ & 0.01 \\
\hline Urinary symptoms & $42.72 \pm 30.41$ & 50 & $30.62 \pm 23.94$ & 44 & $-128.30 \%$ & 0.003 \\
\hline Sexual dysfunction & $28.25 \pm 35.69$ & 50 & $25.28 \pm 33.58$ & 44 & $-110.50 \%$ & 0.784 \\
\hline Miscellaneous & $33.33 \pm 20.73$ & 50 & $18.99 \pm 14.03$ & 44 & $-143.00 \%$ & $<0.0001$ \\
\hline ESS & $9.2 \pm 5.64$ & 49 & $6.93 \pm 5.11$ & 44 & $-124.70 \%$ & 0.012 \\
\hline PSQI & $10.43 \pm 4.02$ & 47 & $8.36 \pm 4.41$ & 42 & $-119.80 \%$ & 0.001 \\
\hline BDI-II & $15.88 \pm 10.46$ & 50 & $10.18 \pm 6.76$ & 44 & $-135.90 \%$ & $<0.0001$ \\
\hline Mood condition & & 50 & & 44 & & 0.021 \\
\hline Major depression (\%) & 52 & & 31.8 & & & \\
\hline Minor depression (\%) & 34 & & 34.1 & & & \\
\hline Subthreshold depression (\%) & 12 & & 22.7 & & & \\
\hline Non-depression (\%) & 2 & & 11.4 & & & \\
\hline KPPS & $40.04 \pm 36.18$ & 48 & $22.6 \pm 21.42$ & 44 & $-143.60 \%$ & $<0.0001$ \\
\hline VAS-PAIN & $4.61 \pm 3.22$ & 49 & $3.67 \pm 2.69$ & 43 & $-120.40 \%$ & 0.071 \\
\hline VAFS_Physical & $4.18 \pm 2.84$ & 49 & $3.64 \pm 2.55$ & 44 & $-112.90 \%$ & 0.293 \\
\hline VAFS-Mental & $3.14 \pm 2.65$ & 49 & $2.45 \pm 2.79$ & 44 & $-121.90 \%$ & 0.118 \\
\hline \multicolumn{7}{|l|}{ QoL and autonomy } \\
\hline PDQ-39SI & $30.07 \pm 17.61$ & 49 & $21.24 \pm 13.48$ & 44 & $-129.40 \%$ & $<0.0001$ \\
\hline Mobility & $34.55 \pm 27.79$ & 49 & $29.09 \pm 26.85$ & 44 & $-115.80 \%$ & 0.037 \\
\hline
\end{tabular}


Table 1 continued

\begin{tabular}{lllllll}
\hline & V1 & $\boldsymbol{N}(\mathbf{V 1})$ & $\mathbf{V 4}$ & $\boldsymbol{N}(\mathbf{V} \mathbf{4})$ & $\boldsymbol{\Delta V 1 - V 4}$ & $\boldsymbol{p}$ \\
\hline Activities of daily living & $26.5 \pm 23.94$ & 49 & $17.8 \pm 17.96$ & 44 & $-132.80 \%$ & $\mathbf{0 . 0 1 4}$ \\
Emotional well-being & $44.3 \pm 29.34$ & 49 & $26.33 \pm 23.01$ & 44 & $-140.60 \%$ & $<\mathbf{0 . 0 0 0 1}$ \\
Stigmatization & $15.82 \pm 22.79$ & 49 & $7.67 \pm 13.13$ & 44 & $-151.50 \%$ & $\mathbf{0 . 0 2 1}$ \\
Social support & $7.48 \pm 16.51$ & 49 & $3.59 \pm 12.63$ & 44 & $-152.00 \%$ & 0.302 \\
Cognition & $27.17 \pm 22$ & 49 & $23.72 \pm 22.49$ & 44 & $-112.70 \%$ & 0.876 \\
Communication & $20.07 \pm 26.73$ & 49 & $12.12 \pm 15.19$ & 44 & $-139.60 \%$ & 0.203 \\
Pain and discomfort & $44.56 \pm 27.35$ & 49 & $33.33 \pm 19.93$ & 44 & $-125.20 \%$ & $\mathbf{0 . 0 1 8}$ \\
ADLS & $81.40 \pm 11.78$ & 50 & $80.91 \pm 16.39$ & 44 & $-10.60 \%$ & 0.845 \\
\hline
\end{tabular}

$p$ values were computed using the Wilcoxon signed-rank or the marginal homogeneity test. The results represent mean \pm SD, median $[\mathrm{p} 25, \mathrm{p} 75]$ or percentage. Domains of the NMSS and PDQ-39SI were expressed as a percentage. Significant values are in bold

ADLS Schwab \& England Activities of Daily Living Scale, BDI Beck Depression Inventory, ESS Epworth Sleepiness Scale, FOGQ Freezing of Gait Questionnaire, $H \mho Y$ Hoenh \& Yahr, KPPS King's PD Pain Scale, NMSS Non-Motor Symptoms Scale, NPI Neuropsychiatric Inventory, PDQ-39SI 39-item Parkinson's Disease Quality of Life Questionnaire Summary Index, PSQI Pittsburgh Sleep Quality Index, UPDRS Unified Parkinson's Disease Rating Scale, VAFS Visual Analog Fatigue Scale, VAS-PAIN Visual Analog Scale-Pain, NA not applicable

frequency of patients with MD was reduced to $31.8 \%(p=0.029)$ (Table 1 and Fig. 4).

A moderate correlation was observed between the change from V1 to V4 in the BDI-II score and the NMSS mood/apathy domain $(r=0.507 ; p<0.0001)$ and the BDI-II and the PDQ-39 emotional well-being domain $(r=0.458 ; p=0.0002)$ scores but not between the NMSS mood/apathy domain and the PDQ39 emotional well-being domain scores $(r=0.272 ; p=0.77)$. A strong correlation was observed between the change from V1 to V4 in the NMSS total score and the NMSS mood/apathy domain score $(r=0.680 ; p<0.0001)$. Other significant correlations are shown in Table 3.

With regard to gender, a significant reduction on BDI-II, NMSS mood/apathy domain, and PDQ-39 emotional well-being domain scores was observed in both men and women, but without differences between groups (a greater reduction in women in the PDQ-39 emotional well-being domain with a trend of significance was observed [ $p=0.092]$; Table 2 in the Supplementary Material). No differences were observed either by age or time from diagnosis except a greater reduction in the NMSS mood/apathy domain score in the subgroup of patients with PD older than 70 years old $(23.49 \pm 21.44$ vs $9.72 \pm 10.87 ; p=0.040)$. No correlations were observed between the change in the BDI-II score from V1 to V4 and age $(r=-0.058 ; p=0.796)$ and mean time from diagnosis of PD $(r=0.094 ; p=0.508)$.

A total of 21 adverse events in 16 patients (32\%) were reported, five of which were severe (not related to safinamide) and six patients abandoned the study for the following reasons: one withdrawal of consent; one discontinuation of treatment after deep brain stimulation procedure; one personal decision due to no effect; three because of an adverse event (two dizziness; one respiratory infection) (Table 3 in the Supplementary Material). The most frequent adverse events were dyskinesia and nausea (6\%), but only one patient discontinued the medication because of an adverse event related to 


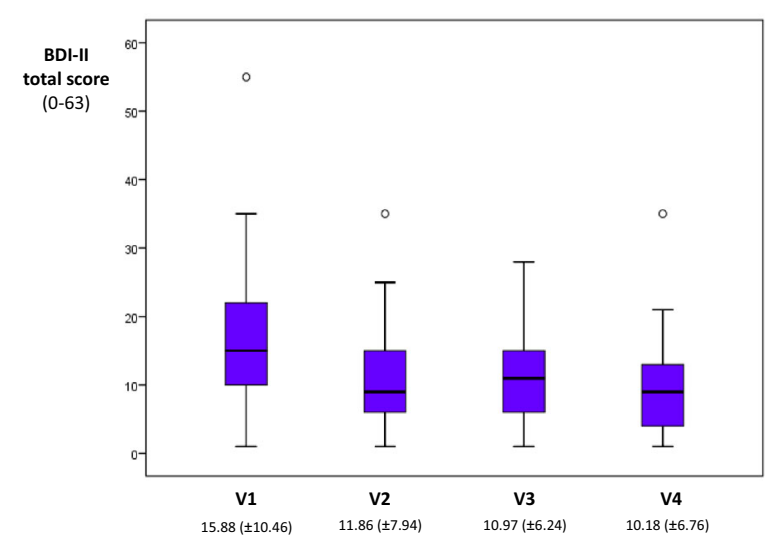

Fig. 1 BDI-II at V1 (baseline), V2 (1 months \pm 7 days), V3 (3 months \pm 15 days) and V4 (6 months \pm 15 days). Compared to V1, a significant reduction was observed at V2, V3 and V4 $(p<0.001)$. Changes were not statistically significant from V2 to V3 and to V4, or from V3 to V4

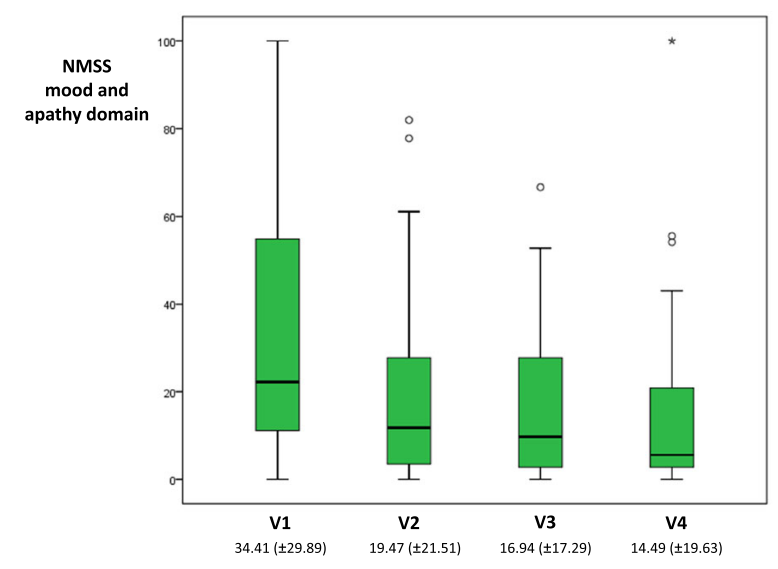

Fig. 2 NMSS mood and apathy domain at V1 (baseline), V2 (1 months \pm 7 days), V3 (3 months \pm 15 days) and V4 (6 months \pm 15 days). Compared to V1, a significant reduction was observed at V1, V2 and V3 $(p<0.001)$. Changes were not statistically significant from V2 to V3 and to V4, or from V3 to V4

safinamide (dizziness). All patients were receiving safinamide $50 \mathrm{mg} /$ day at $\mathrm{V} 2$ except three who were receiving $100 \mathrm{mg} /$ day, whereas all patients were receiving $100 \mathrm{mg} /$ day at $\mathrm{V} 3$ and V4 except one and two cases, respectively, who were receiving $50 \mathrm{mg} /$ day. Only three patients were receiving rasagiline which was withdrawn with a washout period of at least 2 weeks before starting safinamide. At baseline, $38 \%$ of the patients were taking benzodiazepines and 32\%

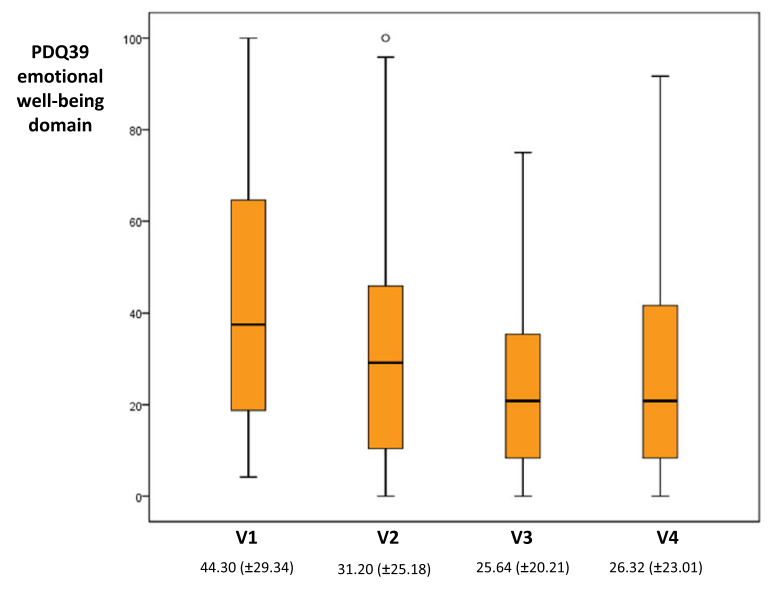

Fig. 3 PDQ39 emotional well-being domain at V1 (baseline), V2 (1 months \pm 7 days), V3 (3 months \pm 15 days) and V4 (6 months \pm 15 days). Compared to V1, a significant reduction was observed at V1, V2 and V3 $(p<0.001)$, and also from V2 to V3 $(p=0.045)$. Changes were not statistically significant from V2 to V4, or from V3 to $\mathrm{V} 4$

any antidepressant agent, the most frequent being a selective serotonin reuptake inhibitor (sertraline, $N=5$; mirtazapine, $N=4$; escitalopram, $N=3$; citalopram, $N=2$; trazodone, $N=2$ ) (Table 1 in the Supplementary Material). Except one patient who added escitalopram to $20 \mathrm{mg} /$ day 93 days after V1, no other changes were conducted in the antidepressant prescriptions. No significant relationship of suffering an adverse event with the use of antidepressants was observed, although they were slightly more frequent in patients receiving an antidepressant agent $(37.5 \%$ vs $26.5 \%, p>0.05)$. Serotonin syndrome was not observed in any patient.

\section{DISCUSSION}

Patients with depression or antidepressant medications were excluded from the first trials of safinamide (studies 015, 016, and SETTLE), but the 18-month extension of study 016 (study 018) observed significant improvements in the Hamilton Rating Scale for Depression scores with the $100 \mathrm{mg}$ dose [14]. However, these patients had low baseline Hamilton Rating Scale for Depression scores. In the SAFINONMOTOR study, patients with PD with severe or very 


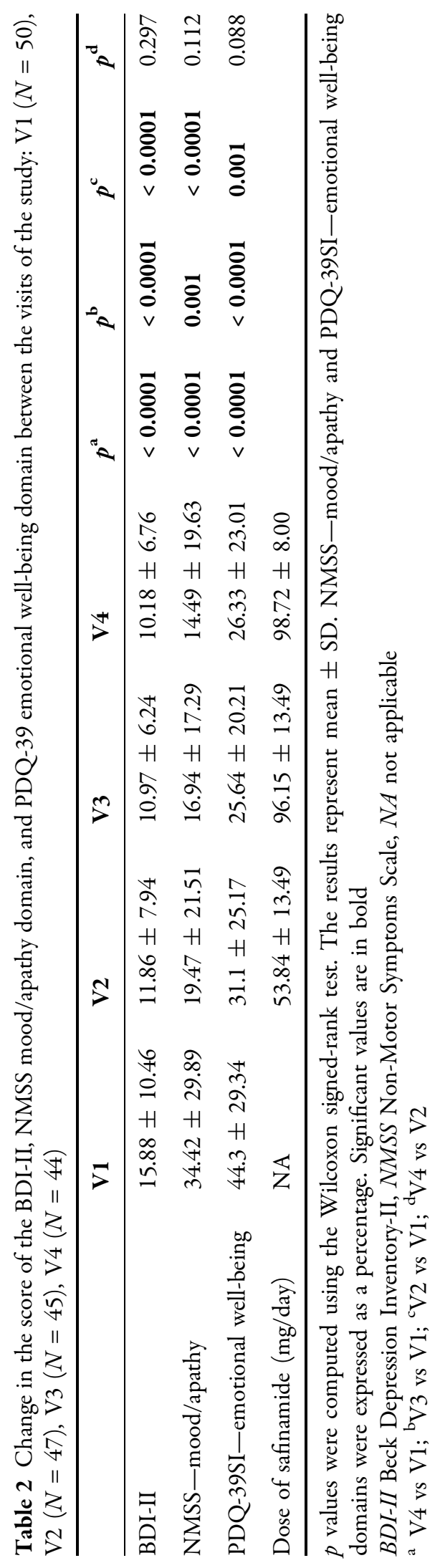




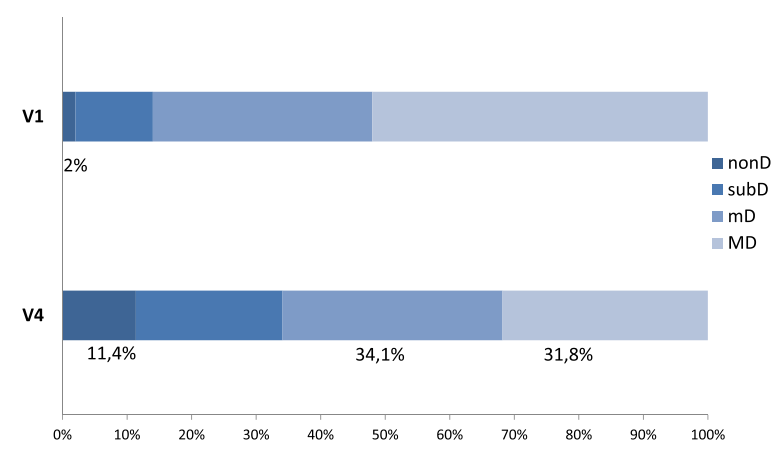

Fig. 4 Proportion of patients with major depression (MD), minor depression ( $\mathrm{mD})$, subthreshold Depression (subD) and non-Depression (nonD) at baseline (V1) and after 6 months (V4). Changes in groups were statistically significant $(p<0.05)$

severe global NMS burden were recruited and baseline evaluations showed a majority of patients suffering depressive symptoms and about a third taking antidepressants. The results of our study demonstrate a sustained positive effect of safinamide on mood and emotional well-being QoL. It is interesting to remark that benefits were observed at V2 with the initial $50 \mathrm{mg}$ dose, and they were sustained until the 6-month follow-up visit after increasing to $100 \mathrm{mg}$ but with no significant differences between 50 and $100 \mathrm{mg}$.

Our results agree with previous data that also show benefits of safinamide on $\operatorname{mood}[4,6]$. Nonetheless, it is important to notice that the existing studies that demonstrate benefits of safinamide on mood are presented with the $100 \mathrm{mg}$ dose, supporting this role on the glutamatergic regulation of safinamide at $100 \mathrm{mg}$. The recent SADNESS study [5] evaluated the effect of safinamide on depression, including patients with doses of $50 \mathrm{mg}$ and $100 \mathrm{mg}$. A significant improvement was observed in the Hamilton Depression Rating Scale for both doses, with no significant differences between 50 and $100 \mathrm{mg}$, although a tendency for better outcomes with the $100 \mathrm{mg}$ treatment was described. However, the authors concluded that there were no statistically significant differences between both doses because the $50 \mathrm{mg}$ group included only 22 subjects. Our study confirms a clear benefit in mood with only $50 \mathrm{mg}$ of safinamide that remains stable with the $100 \mathrm{mg}$ dose. To our knowledge, this is the first time that this benefit is clearly demonstrated at $50 \mathrm{mg}$ dose in a prospective study.

The MAO enzyme has two isoforms (A and B) with different brain distribution and substrates. MAO-B isoform has greater activity in the basal ganglia and does not metabolize noradrenaline or serotonin [15]. MAO-A inhibitors are used as antidepressants because MAO-A metabolizes serotonin. However, it has been proposed that inhibiting MAO-B can indirectly modulate serotonin by the action of dopamine, as the formation of serotonin from tryptophan is dopamine dependent, and that the blocking of dopamine metabolism may be even more effective than the blocking of MAO-A for treating depression $[16,17]$. A dose of $20-40 \mathrm{mg}$ of safinamide can achieve an inhibition of MAO-B over $90 \%$ with no evidence of MAO-A inhibition even with high doses of safinamide [18]. We hypothesize that safinamide-induced improvement of mood in PD depressive symptoms can be modulated by dopaminergic regulation, with a more modest effect of glutamatergic systems. In line with this, pramipexole improved depressive symptoms in patients with PD versus placebo although a direct antidepressant effect has been suggested [19]. Another possible explanation may be that safinamide can regulate glutamate with lower doses than $100 \mathrm{mg}$. It has been suggested that this second action mechanism could explain at least in part the favorable effect of safinamide over some NMS but it is not clear whether the dopaminergic action of safinamide could be more potent than that of other MAO-B inhibitors such as rasagiline or if benefits could be related to its effect involving the glutamatergic system, or both [20]. Previously, treatment with rasagiline did not have significant effects versus placebo on depressive symptoms in patients with PD with moderate depressive symptoms, although post hoc analyses signaled some improvement in patient-rated depression outcomes [21]. To clarify this question, a randomized trial with safinamide at different doses (50 $\mathrm{mg}$ vs 100 vs $>100 \mathrm{mg}$ ) versus placebo and ideally with a rasagiline arm is needed.

Depression is a frequent comorbidity in PD that has a notable negative effect on health- 
Table 3 Correlations between the changes in score of the BDI-II, NMSS mood/apathy domain, and PDQ-39 emotional well-being domain, and changes in other scales from V1 (baseline) to V4 (6 years \pm 15 days)

\begin{tabular}{|c|c|c|c|}
\hline & BDI-II & $\begin{array}{l}\text { NMSS } \\
\text { Mood/apathy }\end{array}$ & $\begin{array}{l}\text { PDQ-39SI } \\
\text { Emotional well-being }\end{array}$ \\
\hline \multicolumn{4}{|l|}{ Motor assessment } \\
\hline UPDRS-III-ON & 0.185 & -10.004 & 0.215 \\
\hline UPDRS-IV & 0.257 & $0.457^{* *}$ & 0.259 \\
\hline FOGQ & 0.093 & 0.187 & -10.035 \\
\hline \multicolumn{4}{|c|}{ Non motor assessment } \\
\hline NMSS total score & $0.513^{*}$ & 0.680 & 0.216 \\
\hline ESS & 0.017 & 0.092 & 0.154 \\
\hline PSQI & 0.022 & $0.352^{* * *}$ & -10.006 \\
\hline BDI-II & NA & $0.507^{*}$ & $0.458^{* *}$ \\
\hline KPPS & $0.432^{* *}$ & 0.253 & 0.268 \\
\hline VAS-PAIN & 0.178 & $0.365^{* * *}$ & 0.028 \\
\hline VAFS_Physical & $0.380^{* * *}$ & $0.389^{* * *}$ & 0.07 \\
\hline VAFS-Mental & $0.580^{*}$ & $0.498^{* *}$ & 0.243 \\
\hline \multicolumn{4}{|l|}{ QoL and autonomy } \\
\hline PDQ-39SI & $0.467^{* *}$ & $0.385^{* * *}$ & $0.522^{*}$ \\
\hline ADLS & -10.121 & -10.289 & -10.116 \\
\hline
\end{tabular}

Spearman correlation test were applied. Significant values are in bold

ADLS Schwab \& England Activities of Daily Living Scale, BDI Beck Depression Inventory, ESS Epworth Sleepiness Scale, FOGQ Freezing of Gait Questionnaire, $H \mho Y$ Hoenh \& Yahr, KPPS King's PD Pain Scale, NMSS Non-Motor Symptoms Scale, NPI Neuropsychiatric Inventory, PDQ-39SI 39-item Parkinson's Disease Quality of Life Questionnaire Summary Index, PSQI Pittsburgh Sleep Quality Index, UPDRS Unified Parkinson's Disease Rating Scale, VAFS Visual Analog Fatigue Scale, VAS-Pain Visual Analog Scale-Pain, $N A$ not applicable

${ }^{*} p<0.0001 ;{ }^{* *} p<0.001 ;{ }^{* *} p<0.05$

related and global QoL of patients with PD, not only major and minor depression, but also subD $[1,22]$. Importantly, we observed that mood improvement 6 months after starting with safinamide correlated with health-related QoL improvement. Systematic reviews of depression in epidemiologic PD studies describe a combined prevalence about 35\% [23], although some publications report frequencies over 50\% $[13,24]$. This prevalence may be higher in patients with disease later stages [22, 25-27] or women $[12,26]$, although depression-type frequency or BDI-II score does not seem to change with disease duration [13]. Furthermore, patients with disease later stages that present motor fluctuations suffer more frequent dysthymia and anxiety symptoms, unrelated to levodopa wearing off or controlled treatment response of fluctuations [28]. In any case, our study corroborates that patients, even without a long disease duration, can present a high NMS burden, mood disorder, and a poor QoL and that it is important to identify this phenotype in clinical practice [29]. Despite this, it is known that only a low proportion of patients with PD with depression receive antidepressant treatments [25] and our findings support this. 
Depression treatment in PD is known to be a challenging issue. Elderly subjects with executive dysfunction have a poorer response to serotonin-reuptake-inhibiting antidepressants [30]. Anxiety is even more frequent than depression in PD [24], and its presence is a predictor of worse response to antidepressants [31]. In a meta-analyses conducted by Huang et al. [32], MAOB-I significantly reduced depressive symptoms, but in the subgroup analysis this decrease was significant only in patients with early PD. Espay et al. [2] analyzed the effect of dopaminergic medications on mood in the PPMI (Parkinson's Progression Markers Initiative) 5-year-data cohort, including levodopa, dopamine agonists (pramipexole, ropinirole, and rotigotine), and the MAOB-I rasagiline and seleginine. They concluded that dopaminergic medications showed no direct effect on mood in patients with early PD, although they observed a non-significant improvement trend in MAOB-I treatment $(-0.35 ; 95 \%$ CI $-0.73,0.04 ; p=0.08)$. Interestingly, cognitive function assessed by the Montreal Cognitive Assessment (MoCA) influenced the effect of medications on mood: dopamine agonists in patients with worse MoCA scores produced a more pronounced worsening of depression scores $(p=0.005)$ while MAOB-I had a moderating effect on depression scales $(p=0.03)$. This fact supports a non-dopaminergic mechanism of depressive symptoms. Therefore, considering the poor response of other dopaminergic treatments in mood as previously mentioned, safinamide may be an option to contemplate when adjusting dopaminergic treatment in patients with PD with depressive symptoms [5]. In addition, a major concern when using safinamide in patients with PD with depression is concomitant antidepressants but our results suggest that safinamide is safe and well tolerated even in this type of PD population.

Our study has important limitations. First, we do not have a placebo arm group to compare differences. However, the effect of the treatment was stable and sustained at the 6-month visit. We consider that this stable lapse of time supports the effect of the treatment, although a possible bias cannot be completely excluded.
Second, our inclusion criteria required patients with a severe or very severe NMS burden, so we cannot conclude that these results can be extrapolated to all subjects with PD. Third, we present a relatively small sample, although being able to demonstrate significant differences with a small sample may also reflect a stronger effect of the treatment. Fourth, patients with PD taking an antidepressant agent were included and one patient started with escitalopram after the baseline visit. However, in practice, many patients with $\mathrm{PD}$ receive antidepressant agents and have depressive symptoms; it of interest that safinamide can be associated with antidepressant agents. Lastly, the COVID-19 pandemic affected the last months of follow-up in some patients, which could have influenced their mood, and four V4 visits had to be conducted by telephone interview.

\section{CONCLUSION}

Safinamide can be useful in the treatment of depression in PD and improves QoL. In this study we demonstrate a significant benefit in BDI-II, as well as in the NMSS mood/apathy domain and the PDQ-39 emotional well-being domain scores with safinamide at 6 months, with no significant increase in adverse events in patients who already receive antidepressants. Further studies including double-blind longer follow-ups are required to analyze the role of safinamide in the regulation of mood in PD.

\section{ACKNOWLEDGEMENTS}

We would like to thank all patients and their caregivers who collaborated in this study. Many thanks also to Fundación Española de Ayuda a la Investigación en Parkinson y otras Enfermedades Neurodegenerativas (Curemos el Parkinson) (https://curemoselparkinson.org/), Leon Research (https://leonresearch.com/es/ inicio-leon-research-ensayos-clinicos-espanaitalia-portugal/), and Zambon España. 
Funding. The present study is a study promoted by an independent researcher (Diego Santos García). Zambon Spain has financed its expenses and also the journal's (ADTH) Rapid Service and Open Access fees.

Authorship. All named authors meet the International Committee of Medical Journal Editors (ICMJE) criteria for authorship for this article, take responsibility for the integrity of the work as a whole, and have given their approval for this version to be published.

Authors' Contributions. All named authors meet the International Committee of Medical Journal Editors (ICMJE) criteria for authorship for this article, take responsibility for the integrity of the work as a whole, and have given their approval for this version to be published. RYB: review and critique; recruitment and/or evaluation of participants. $\mathrm{MICH}$ : review and critique; recruitment and/or evaluation of participants. ICL: review and critique; recruitment and/or evaluation of participants. JMPG: review and critique; recruitment and/or evaluation of participants. MGAL: review and critique; recruitment and/or evaluation of participants. MJGP: recruitment and/or evaluation of participants. CMM: review and critique; organization of material and data. CML: initial draft of the manuscript; recruitment and/or evaluation of participants. DSG: conception, organization, and execution of the project; review and critique. All authors have read and agreed to the published version of the manuscript.

Disclosures. Carmen M. Labandeira has received honoraria for educational presentations and advice service by Abbvie, Italfarmaco, Zambon, and Bial; Maria G. Alonso Losada has received honoraria for educational presentations and advice service by Zambon and Bial; Rosa Yáñez Baña has received honoraria for educational presentations by Teva, Bial, and Zambon; Maria I. Cimas Hernando has received honoraria for educational presentations and advice service by KRKA, Italfarmaco, Teva, Zambon, and Bial; Iria Cabo López has received honoraria for educational presentations and advice service by Abbvie, Zambo, and Bial; Jose
M. Paz González has received honoraria for educational presentations and/or advice service by UCB Pharma, Lundbeck, KRKA, and Zambon; Maria J. González Palmás: None; Cristina Martínez Miró: None; Diego Santos García has received honoraria for educational presentations and advice service by Abbvie, UCB Pharma, Lundbeck, KRKA, Zambon, Bial, Italfarmaco, and Teva.

Compliance with Ethics Guidelines. This study was approved by the by the Comite de Ética de la Investigación Clínica de Galicia from Spain (2018-052; 28/FEB/2019) and all participants gave their written informed consent before inclusion in the study. SAFINONMOTOR was classified by the AEMPS (Agencia Española del Medicamento y Productos Sanitarios) as a post-authorization prospective follow-up study with the code DSG-SAF-2018-01. This study was performed in accordance with the Helsinki Declaration of 1964, and its later amendments, and all subjects provided informed consent to participate in the study.

Data Availability. The protocol and the statistical analysis plan are available on request. Deidentified participant data are not available for legal and ethical reasons.

Open Access. This article is licensed under a Creative Commons Attribution-NonCommercial 4.0 International License, which permits any non-commercial use, sharing, adaptation, distribution and reproduction in any medium or format, as long as you give appropriate credit to the original author(s) and the source, provide a link to the Creative Commons licence, and indicate if changes were made. The images or other third party material in this article are included in the article's Creative Commons licence, unless indicated otherwise in a credit line to the material. If material is not included in the article's Creative Commons licence and your intended use is not permitted by statutory regulation or exceeds the permitted use, you will need to obtain permission directly from the copyright holder. To view a copy of this licence, visit http://creativecommons.org/licenses/by$\mathrm{nc} / 4.0 /$. 


\section{REFERENCES}

1. Santos-García D, de Deus FT, Suárez Castro E, et al. Quality of life and non-motor symptoms in Parkinson's disease patients with subthreshold depression. J Neurol Sci. 2020;418: 117109. https:// doi.org/10.1016/j.jns.2020.117109.

2. Espay AJ, Foster ED, Coffey CS, et al. Lack of independent mood-enhancing effect for dopaminergic medications in early Parkinson's disease. J Neurol Sci. 2019;402:81-5. https://doi.org/10.1016/j.jns. 2019.05.009.

3. Giossi R, Carrara F, Mazzari M, et al. Overall efficacy and safety of safinamide in Parkinson's disease: a systematic review and a meta-analysis. Clin Drug Investig. 2021;41:321-39. https://doi.org/10.1007/ s40261-021-01011-y.

4. Bianchi LME, Riboldazzi G, Mauri M, Versino M. Efficacy of safinamide on non-motor symptoms in a cohort of patients affected by idiopathic Parkinson's disease. Neurol Sci. 2019;40:275-9.

5. Peña E, Borrue C, Mata $M$, et al. Impact of SAfinamide on Depressive Symptoms in Parkinson's Disease Patients (SADness-PD Study): a multicenter retrospective study. Brain Sci. 2021;11:232.

6. Cattaneo C, Muller T, Bonizzonic E, Lazzeria G, Kottakisa I, Keywoodd C. Long-term effects of safinamide on mood fluctuations in Parkinson's disease. J Parkinsons Dis. 2017;7:629-34.

7. Santos García D, Labandeira Guerra C, Yáñez Baña $\mathrm{R}$, et al. Safinamide improves non-motor symptoms burden in Parkinson's disease: an open-label prospective study. Brain Sci. 2021;11:316.

8. Daniel S, Lees A. Parkinson's Disease Society Brain Bank, London: overview and research. J Neural Transm Suppl. 1993;39:165-72.

9. Dubois B, Burn D, Goetz C, et al. Diagnostic procedures for Parkinson's disease dementia: recommendations from the Movement Disorder Society task force. Mov Disord. 2007;22:2314-24.

10. American Psychiatric Association. Diagnostic and statical manual of mental disorders. 4th ed. Washington, DC: American Psychiatric Association; 1994.

11. Judd LL, Rapaport MH, Paulus MP, Brown JL. Subsyndromal symptomatic depression: a new mood disorder? J Clin Psychiatry. 1994;55(Suppl):18-28.

12. Martinez-Martin P, Pecurariu CF, Odin P, et al. Gender-related differences in the burden of non- motor symptoms in Parkinson's disease. J Neurol. 2012;259:1639-47.

13. Santos-García D, De Deus FT, Cores BC, et al. Mood in Parkinson's disease: from early- to late-stage disease. Int J Geriatr Psychiatry. 2021;36:627-46.

14. Borgohain R, Szasz J, Stanzione P, Meshram C, Bhatt $\mathrm{MH}$. Two-year, randomized, controlled study of safinamide as add-on to levodopa in mid to late Parkinson's disease. Mov Disord. 2014;29:1273-80.

15. Collins GG, Sandler M, Williams ED, Youdim MB. Multiple forms of human brain mitochondrial monoamine oxidase. Nature. 1970;28:817-20.

16. Youdim MBH. Monoamine oxidase inhibitors, and iron chelators in depressive illness and neurodegenerative diseases. J Neural Transm. 2018;125: 1719-33. https://doi.org/10.1007/s00702-0181942-9.

17. Youdim $\mathrm{MBH}$, Bakhle YS. Monoamine oxidase: isoforms and inhibitors in Parkinson's disease and depressive illness. Br J Pharmacol. 2006;147: S287-96.

18. Marzo A, Dal Bo L, Ceppi Monti N, et al. Pharmacokinetics and pharmacodynamics of safinamide, a neuroprotectant with antiparkinsonian and anticonvulsant activity. Pharmacol Res. 2004;50:77-85.

19. Barone P, Poewe W, Albrecht S, et al. Pramipexole for the treatment of depressive symptoms in patients with Parkinson's disease: a randomised, double-blind, placebo-controlled trial. Lancet Neurol. 2010;9:573-80.

20. Stocchi F, Torti M. Adjuvant therapies for Parkinson's disease: critical evaluation of safinamide. Drug Des Devel Ther. 2016;10:609-18.

21. Barone P, Santangelo G, Morgante L, et al. A randomized clinical trial to evaluate the effects of rasagiline on depressive symptoms in non-demented Parkinson's disease patients. Eur J Neurol. 2015;22:1184-91.

22. Su W, Liu H, Jiang Y, et al. Correlation between depression and quality of life in patients with Parkinson's disease. Clin Neurol Neurosurg. 2021;202: 106523. https://doi.org/10.1016/j. clineuro.2021.106523.

23. Reijnders JSAM, Ehrt U, Weber WEJ, Aarsland D, Leentjens AFG. A systematic review of prevalence studies of depression in Parkinson's disease. Mov Disord. 2008;23:183-9.

24. Rodriguez-Blazquez C, Schrag A, Rizos A, Chaudhuri KR, Martinez-Martin P, Weintraub D. Prevalence of non-motor symptoms and non-motor 
fluctuations in Parkinson's disease using the MDSNMS. Mov Disord Clin Pr. 2020;8:231-9.

25. Van Der Hoek TC, Bus BAA, Matui P, Van Der Marck MA, Esselink RA, Tendolkar I. Prevalence of depression in Parkinson's disease: effects of disease stage, motor subtype and gender. J Neurol Sci. 2011;310:220-4. https://doi.org/10.1016/j.jns. 2011.07.007.

26. Rojo A, Aguilar M, Garolera MT, Cubo E, Navas I, Quintana S. Depression in Parkinson's disease: clinical correlates and outcome. Parkinsonism Relat Disord. 2003;10:23-8.

27. Sagna A, Gallo J, Pontone GM, Sciences B, Hopkins J. Systematic review of factors associated with depression and anxiety disorders among older adults with Parkinson's disease. Parkinsonism Relat Disord. 2014;20:708-15.

28. Leentjens AFG, Dujardin K, Marsh L, Martinezmartin P, Richard IH, Starkstein SE. Anxiety and motor fluctuations in Parkinson's disease: a crosssectional observational study. Parkinsonism Relat
Disord. 2012;18:1084-8. https://doi.org/10.1016/j. parkreldis.2012.06.007.

29. Santos García D, De Deus FT, et al. Staging Parkinson's disease combining motor and non-motor symptoms correlates with disability and quality of life. Parkinsons Dis. 2021. https://doi.org/10.1155/ 2021/8871549 (eCollection 2021).

30. Alexopoulos GS, Kiosses DN, Heo M, Murphy CF, Shanmugham B, Gunning-dixon F. Executive dysfunction and the course of geriatric depression. Biol Psychiatry. 2005;58:204-10.

31. Moonen AJH, Wijers A, Leentjens AFG, et al. Severity of depression and anxiety are predictors of response to antidepressant treatment in Parkinson's disease. Parkinsonism Relat Disord. 2014;20:644-6. https://doi.org/10.1016/j.parkreldis.2014.02.025.

32. Huang YH, Chen JH, Loh EW, Chan L, Hong CT. The effect of monoamine oxidase-B inhibitors on the alleviation of depressive symptoms in Parkinson's disease: meta-analysis of randomized controlled trials. Ther Adv Psychopharmacol. 2021;11: $1-9$. 